\title{
FURTHER EVALUATION OF A STOCHASTIC MODEL APPLIED TO MONOENERGETIC SPACE-TIME NUCLEAR REACTOR KINETICS
}

\author{
PHAM NHU VIET HA ${ }^{1 \dagger}$ and JONG KYUNG KIM ${ }^{1 *}$ \\ ${ }^{1}$ Department of Nuclear Engineering, Hanyang University \\ 17 Haengdang-dong, Seongdong-gu, Seoul 133-791, KOREA \\ † Present address: Korea Atomic Energy Research Institute \\ 1045 Daedeok-daero, Yuseong-gu, Daejeon 305-353, KOREA \\ *Corresponding author. E-mail : jkkim1@hanyang.ac.kr
}

Received October 01, 2010

Accepted for Publication June 01, 2011

In a previous study, the stochastic space-dependent kinetics model (SSKM) based on the forward stochastic model in stochastic kinetics theory and the Itô stochastic differential equations was proposed for treating monoenergetic space-time nuclear reactor kinetics in one dimension. The SSKM was tested against analog Monte Carlo calculations, however, for exemplary cases of homogeneous slab reactors with only one delayed-neutron precursor group. In this paper, the SSKM is improved and evaluated with more realistic and complicated cases regarding several delayed-neutron precursor groups and heterogeneous slab reactors in which the extraneous source or reactivity can be introduced locally. Furthermore, the source level and the initial conditions will also be adjusted to investigate the trends in the variances of the neutron population and fission product levels across the reactor. The results indicate that the improved SSKM is in good agreement with the Monte Carlo method and show how the variances in population dynamics can be controlled.

KEYWORDS : Space-time Dependent, Stochastic Kinetics, Forward Stochastic Model, Itô Stochastic Differential Equation, Heterogeneous Reactor, Dynamic Fluctuation

\section{INTRODUCTION}

To thoroughly understand the kinetic behavior of a reactor, it is necessary to interpret the equations for the response of prompt and delayed neutron populations to changes in reactivity $[1,2,3]$. This problem is mathematically complex because the neutron population in a reactor is actually a function of both space (i.e., position in the core) and time $[4,5,6]$. In addition to treating deterministic behaviors, random fluctuations in population dynamics should also be appropriately assessed for various practical situations such as low reactor power levels $[7,8,9]$.

In a previous study [10], a simplified stochastic model (called the stochastic space-dependent kinetics model or SSKM) based on the forward stochastic model (FSM) in stochastic kinetics theory [4,5] and the Itô stochastic differential equations $[11,12,13]$ was proposed for the analysis of monoenergetic space-time nuclear reactor kinetics in one dimension. First, the FSM equations to determine the mean values of neutron and delayed-neutron precursor populations were considered as deterministic without taking into account their variances and co-variances. Second, the system of interest was randomized again in the light of the Itô stochastic differential equations in order to derive the SSKM, thereby enabling the computation of the expected values of neutron and cumulative fission densities as well as their standard deviations. Thus, the SSKM may be usefully applied in the treatment of multiregion reactors compared with other methods due to its simplicity and feasibility in modeling the random spacetime behavior of the population dynamics. Nevertheless, the SSKM was tested against analog Monte Carlo calculations for exemplary cases of uniform slab reactors with just one delayed-neutron precursor group. Therefore, further work is still needed to enhance the model and to evaluate its applicability in nuclear reactor transient analysis.

In the present study, the SSKM is improved and evaluated with more realistic and complicated cases including $M$ delayed-neutron precursor groups and 
heterogeneous slab reactors where the extraneous source or reactivity can be introduced locally. Additionally, the source level and the initial conditions are adjusted to recognize the trends in the variances, i.e., the random fluctuations of the neutron population and fission product levels across the reactor. The numerical results demonstrate that the improved SSKM is in good agreement with the Monte Carlo method and show how the variances in population dynamics can be controlled.

\section{IMPROVEMENT OF THE SSKM TO TREAT M DELAYED-NEUTRON PRECURSOR GROUPS}

\subsection{Overview of the SSKM for One Precursor Group}

In the forward stochastic model (FSM) of the stochastic kinetics theory, the spatial domain of a reactor is partitioned into $I$ space cells, and the energy range is partitioned into $G$ energy cells $[4,5]$. Subject to this partitioning, the state of the reactor is defined by the set of numbers

$$
N=\left\{n_{i g} c_{i m}\right\}, i=1,2, \ldots, I ; g=1,2, \ldots, G ; m=1,2, \ldots, M \text {, }
$$

where $n_{i g}$ is the number of neutrons in space cell $i$ and energy cell $g$, and $c_{i m}$ is the number of $m$-type delayed neutron precursors in space cell $i$.

Starting with the concept of reactor transition probability and its probability generating function, the FSM equations for mean value of neutron and precursor distribution are formed as follows:

$$
\begin{aligned}
\frac{\partial \bar{n}_{i g}(t)}{\partial t}=S_{i g}(t)-\left[\Lambda_{c i g}(t)\right. & \left.+\Lambda_{s i g}(t)+\Lambda_{f i g}(t)\right] \bar{n}_{i g}(t) \\
& +\sum_{g^{\prime}=1}^{G} \Lambda_{s i g^{\prime}}(t) K_{i}^{g^{\prime} g} \bar{n}_{i g^{\prime}}(t) \\
& +\chi_{p}^{g} \sum_{g^{\prime}=1}^{G} \bar{v}_{p}^{g^{\prime}} \Lambda_{f i g^{\prime}}(t) \bar{n}_{i g^{\prime}}(t)+\sum_{m=1}^{M} \chi_{m}^{g} \lambda_{m} \bar{c}_{i m}(t) \\
& +\sum_{i^{\prime}=1}^{I}\left[l_{i^{\prime} i}^{g}(t) \bar{n}_{i^{\prime} g}(t)-l_{i i^{\prime}}^{g}(t) \bar{n}_{i g}(t)\right], \\
\frac{\partial \bar{c}_{i m}(t)}{\partial t}= & -\lambda_{m} \bar{c}_{i m}(t)+\beta_{m}^{\prime} \sum_{g=1}^{G} \bar{v}_{p}^{g} \Lambda_{f i g}(t) \bar{n}_{i g}(t),
\end{aligned}
$$

where

$S_{i g}(t)$ is the neutron source rate in space cell $i$ and energy cell $g$,

$\Lambda_{c i g}(t)$ is the capture frequency per neutron in space cell $i$ and energy cell $g$,

$\Lambda_{\text {sig }}(t)$ is the scattering frequency per neutron in space cell $i$ and energy cell $g$,

$\Lambda_{\text {fig }}(t)$ is the fission frequency per neutron in space cell $i$ and energy cell $g\left(\Lambda_{f i g}=v^{g} \Sigma_{f}^{g}\right.$ where $v^{g}$ is the neutron speed, and $\Sigma_{f}^{g}$ is the macroscopic fission cross section), $K^{g g^{\prime}}$ is the probability that a scattering event which occurred in energy cell $g$ transfers a neutron to energy cell $g^{\prime}$, $\chi_{p}^{g}$ is the probability that a prompt neutron produced by fission has energy within energy cell $g$,

$\chi_{m}^{g}$ is the probability that a delayed neutron produced by $m$-type precursor decay has energy within energy cell $g$, $\lambda_{m}$ is the decay constant for precursor type $m$,

$\beta_{m}^{\prime}$ is the average ratio of the number of $m$-type precursors to the number of prompt neutrons produced in a fission $\left(\beta^{\prime}=\Sigma_{m} \beta_{m}^{\prime}\right)$,

$l_{i i^{\prime}}^{g}(t)$ represents the frequency per neutron at which neutrons in space cell $i$ and energy cell $\mathrm{g}$ will diffuse into space cell $i^{\prime}$ (without a change in energy), $v_{p}^{g}=(1-\beta) \bar{v}^{g}$, where $\bar{v}^{g}$ is the average number of neutrons (prompt and delayed) per fission induced by a neutron in energy $g$, and $\beta^{\prime}=\beta /(1-\beta)$, where $\beta$ is the total delayed neutron fraction.

As shown in the previous study, the stochastic spacedependent kinetics model (SSKM) based on the FSM equations (1) and (2) and the Itô stochastic differential equations was proposed for one spatial dimension, one energy cell, and one precursor as follows:

$$
\begin{aligned}
\frac{d}{d t}\left[\begin{array}{l}
\bar{n}_{i}(t) \\
\bar{c}_{i 1}(t)
\end{array}\right]= & \hat{A}\left[\begin{array}{l}
\bar{n}_{i}(t) \\
\bar{c}_{i 1}(t)
\end{array}\right]+\left[\begin{array}{c}
q_{i}(t) \\
0
\end{array}\right] \\
& +\hat{B}^{\frac{1}{2}} \frac{d \vec{W}(t)}{d t}, i=1,2, \ldots, I
\end{aligned}
$$

where

$$
\begin{gathered}
\hat{A}=\left[\begin{array}{ll}
-d+b\left(\bar{v}_{p}-1\right) & \lambda_{1} \\
\beta_{1}^{\prime} \bar{v}_{p} b & -\lambda_{1}
\end{array}\right], \\
\hat{B}=\left[\begin{array}{l}
\left(d+b\left(\bar{v}_{p}-1\right)^{2} \bar{n}_{i}(t)+\lambda_{1} \bar{c}_{i 1}(t)+Q_{i}(t)\right. \\
\beta_{1}^{\prime} \bar{v}_{p}\left(\bar{v}_{p}-1\right) b \bar{n}_{i}(t)-\lambda_{1} \bar{c}_{i 1}(t) \\
\left.\beta_{1}^{\prime} \bar{v}_{p}\left(\bar{v}_{p}-1\right) b \bar{n}_{i}(t)-\lambda_{1} \bar{c}_{i 1}(t)\right], \\
\left(\beta_{1}^{\prime} \bar{v}_{p}\right)^{2}+\lambda_{1} \bar{c}_{i 1}(t)
\end{array}\right] \\
b=\frac{\left(\bar{v}_{p}-1\right) \Lambda_{f i}(t)}{\bar{v}_{p}-1}=\Lambda_{f i}(t), \\
d \equiv \Lambda_{c i}(t), \\
q_{i}(t)=\sum_{i^{\prime}=i-1}^{i+1}\left[l_{i^{\prime} i}(t) \bar{n}_{i^{\prime}}(t)-l_{i i^{\prime}}(t) \bar{n}_{i}(t)\right]+S_{i}(t), \\
Q_{i}(t)=l_{i+1 i}(t) \bar{n}_{i+1}(t)+l_{i-1 i}(t) \bar{n}_{i-1}(t) \\
+\left[l_{i i+1}(t)+l_{i i-1}(t)\right] \bar{n}_{i}(t)+S_{i}(t),
\end{gathered}
$$

and

$$
\vec{W}(t)=\left[\begin{array}{l}
W_{1}(t) \\
W_{2}(t)
\end{array}\right] \text { is the two-dimensional Wiener process [10] }
$$


Eq. (3) is the stochastic space-dependent kinetics model (SSKM) for one spatial dimension, one energy cell, and one delayed-neutron precursor group. To deal with $M$ precursor groups, the SSKM will be improved, as presented in the following section.

\subsection{Improvement of the SSKM to Treat M Precursor Groups}

For one spatial dimension and one energy cell $(G=1)$, the FSM equations (1) and (2) for the mean values of neutron and precursor populations become

$$
\begin{gathered}
\frac{\partial \bar{n}_{i}(t)}{\partial t}=-\Lambda_{c i}(t) \bar{n}_{i}(t)+\left(\bar{v}_{p}-1\right) \Lambda_{f i}(t) \bar{n}_{i}(t)+\sum_{m=1}^{M} \lambda_{m} \bar{c}_{i m}(t) \\
+\sum_{i^{\prime}=i-1}^{i+1}\left[l_{i^{\prime} i}(t) \bar{n}_{i^{\prime}}(t)-l_{i^{\prime}}(t) \bar{n}_{i}(t)\right]+S_{i}(t) \\
\frac{\partial \bar{c}_{i m}(t)}{\partial t}=-\lambda_{m} \bar{c}_{i m}(t)+\beta_{m}^{\prime} \bar{v}_{p} \Lambda_{f i}(t) \bar{n}_{i}(t)
\end{gathered}
$$

where an assumption was made that neutrons can only diffuse between successive cells.

In accordance with the previous study [10], it is crucial to consider the changes in the neutron and delayed-neutron precursor populations in a very small time interval, $\Delta t$, where the probability of more than one event occurring during time $\Delta t$ is negligible. During time $\Delta t$, there are $M+6$ different possibilities for an event to occur. Let $\left[\Delta \bar{n}_{i}(t)\right.$, $\left.\Delta \bar{c}_{i 1}(t), \Delta \bar{c}_{i 2}(t), \ldots, \Delta \bar{c}_{i M}(t)\right]^{T}$ be the change in the populations $\bar{n}_{i}(t)$ and $\bar{c}_{i m}(t)(m=1,2, \ldots, M)$ in time $\Delta t$. Also, it is assumed that the changes are approximately normally distributed. The $M+6$ possibilities for $\left[\Delta \bar{n}_{i}(t), \Delta \bar{c}_{i 1}(t)\right.$, $\left.\Delta \bar{c}_{i 2}(t), \ldots, \Delta \bar{c}_{i M}(t)\right]^{T}$

are

$$
\begin{aligned}
& {\left[\begin{array}{c}
\Delta \bar{n}_{i}(t) \\
\Delta \bar{c}_{i 1}(t) \\
\Delta \bar{c}_{i 2}(t) \\
\ldots \\
\Delta \bar{c}_{i M}(t)
\end{array}\right]_{1}\left[\begin{array}{c}
-1 \\
0 \\
0 \\
\ldots \\
0
\end{array}\right], \quad\left[\begin{array}{c}
\Delta \bar{n}_{i}(t) \\
\Delta \bar{c}_{i 1}(t) \\
\Delta \bar{c}_{i 2}(t) \\
\ldots \\
\Delta \bar{c}_{i M}(t)
\end{array}\right]_{2}=\left[\begin{array}{c}
\bar{v}_{p}-1 \\
\beta_{1}^{\prime} \bar{v}_{p} \\
\beta_{2}^{\prime} \bar{v}_{p} \\
\ldots \\
\beta_{M}^{\prime} \bar{v}_{p}
\end{array}\right],} \\
& {\left[\begin{array}{c}
\Delta \bar{n}_{i}(t) \\
\Delta \bar{c}_{i 1}(t) \\
\Delta \bar{c}_{i 2}(t) \\
\ldots \\
\Delta \bar{c}_{i M}(t)
\end{array}\right]_{3}\left[\begin{array}{c}
1 \\
-1 \\
0 \\
\ldots \\
0
\end{array}\right],} \\
& {\left[\begin{array}{c}
\Delta \bar{n}_{i}(t) \\
\Delta \bar{c}_{i 1}(t) \\
\Delta \bar{c}_{i 2}(t) \\
\ldots \\
\Delta \bar{c}_{i M}(t)
\end{array}\right]_{4}=\left[\begin{array}{r}
1 \\
0 \\
-1 \\
\ldots \\
0
\end{array}\right], \ldots,\left[\begin{array}{c}
\Delta \bar{n}_{i}(t) \\
\Delta \bar{c}_{i 1}(t) \\
\Delta \bar{c}_{i 2}(t) \\
\ldots \\
\Delta \bar{c}_{i M}(t)
\end{array}\right]_{M+2}=\left[\begin{array}{r}
1 \\
0 \\
0 \\
\ldots \\
-1
\end{array}\right],}
\end{aligned}
$$

$$
\begin{aligned}
& {\left[\begin{array}{c}
\Delta \bar{n}_{i}(t) \\
\Delta \bar{c}_{i 1}(t) \\
\Delta \bar{c}_{i 2}(t) \\
\ldots \\
\Delta \bar{c}_{i M}(t)
\end{array}\right]_{M+3}=\left[\begin{array}{c}
1 \\
0 \\
0 \\
\ldots \\
0
\end{array}\right],} \\
& {\left[\begin{array}{c}
\Delta \bar{n}_{i}(t) \\
\Delta \bar{c}_{i 1}(t) \\
\Delta \bar{c}_{i 2}(t) \\
\ldots \\
\Delta \bar{c}_{i M}(t)
\end{array}\right]_{M+4}=\left[\begin{array}{c}
1 \\
0 \\
0 \\
\ldots \\
0
\end{array}\right], \quad\left[\begin{array}{c}
\Delta \bar{n}_{i}(t) \\
\Delta \bar{c}_{i 1}(t) \\
\Delta \bar{c}_{i 2}(t) \\
\ldots \\
\Delta \bar{s}_{i M}(t)
\end{array}\right]_{M+5}=\left[\begin{array}{c}
-1 \\
0 \\
0 \\
\ldots \\
0
\end{array}\right],} \\
& {\left[\begin{array}{c}
\Delta \bar{n}_{i}(t) \\
\Delta \bar{c}_{i 1}(t) \\
\Delta \bar{c}_{i 2}(t) \\
\cdots \\
\Delta \bar{c}_{i M}(t)
\end{array}\right]_{M+6}=\left[\begin{array}{c}
1 \\
0 \\
0 \\
\ldots \\
0
\end{array}\right] .}
\end{aligned}
$$

Subsequently, the probabilities of these events are

$$
\begin{aligned}
& P_{1}=d \bar{n}_{i}(t) \Delta t=\Lambda_{c i}(t) \bar{n}_{i}(t) \Delta t, \\
& P_{2}=b \bar{n}_{i}(t) \Delta t=\Lambda_{f i}(t) \bar{n}_{i}(t) \Delta t, \\
& P_{3}=\lambda_{1} \bar{c}_{i 1}(t) \Delta t, \\
& P_{4}=\lambda_{2} \bar{c}_{i 2}(t) \Delta t, \ldots, \\
& P_{M+2}=\lambda_{M} \bar{c}_{i M}(t) \Delta t, \\
& P_{M+3}=l_{i-1 i}(t) \bar{n}_{i-1}(t) \Delta t, \\
& P_{M+4}=l_{i+1 i}(t) \bar{n}_{i+1}(t) \Delta t, \\
& P_{M+5}=\left[l_{i i+1}(t)+l_{i i-1}(t)\right] \bar{n}_{i}(t) \Delta t, \\
& P_{M+6}=S_{i}(t) \Delta t,
\end{aligned}
$$

where it is assumed that the extraneous source produces neutrons randomly following a Poisson process with intensity $S_{i}(t)$.

The similar approach adopted from the previous study [10] gives the following Itô stochastic differential equation:

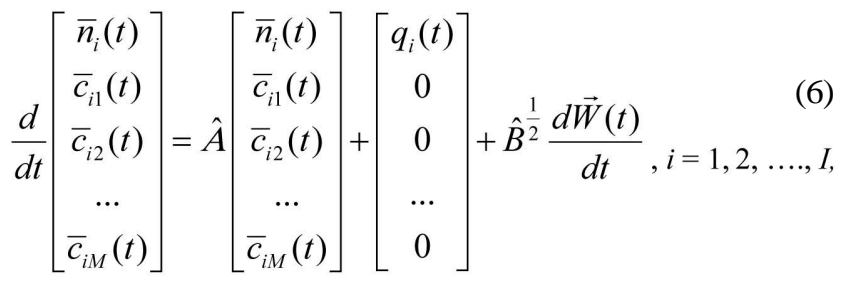


where

$$
\begin{aligned}
& \hat{A}=\left[\begin{array}{lrrrr}
-d+b\left(\bar{v}_{p}-1\right) & \lambda_{1} & \lambda_{2} & \ldots & \lambda_{M} \\
\beta_{1}^{\prime} \bar{v}_{p} b & -\lambda_{1} & 0 & \ldots & 0 \\
\beta_{2}^{\prime} \bar{v}_{p} b & 0 & -\lambda_{2} & \ldots & 0 \\
\ldots & & & & \\
\beta_{M}^{\prime} \bar{v}_{p} b & 0 & 0 & \ldots & -\lambda_{M}
\end{array}\right], \\
& \hat{B}=\left[\begin{array}{ccccc}
\xi & a_{1} & a_{2} & \ldots & a_{M} \\
a_{1} & r_{1} & b_{2,3} & \ldots & b_{2, M} \\
a_{2} & b_{3,2} & r_{2} & \ldots & b_{3, M} \\
\ldots & & & & \\
a_{M-1} & b_{M-1,2} & \ldots & r_{M-1} & b_{M-1, M} \\
a_{M} & b_{M, 2} & \ldots & b_{M, M-1} & r_{M}
\end{array}\right],
\end{aligned}
$$$$
b \equiv \Lambda_{f i}(t),
$$$$
d \equiv \Lambda_{c i}(t)
$$$$
\xi=\left(d+b\left(\bar{v}_{p}-1\right)^{2}\right) \bar{n}_{i}(t)+\sum_{m=1}^{M} \lambda_{m} \bar{c}_{i m}(t)+Q_{i}(t),
$$$$
a_{m}=\beta_{m}^{\prime} \bar{v}_{p} b\left(\bar{v}_{p}-1\right) \bar{n}_{i}(t)-\lambda_{m} \bar{c}_{i m}(t),
$$$$
b_{m, m^{\prime}}=\left(\beta_{m}^{\prime} \bar{v}_{p}\right)\left(\beta_{m^{\prime}}^{\prime}, \bar{v}_{p}\right) b \bar{n}_{i}(t) \text {, }
$$$$
r_{m}=\left(\beta_{m}^{\prime} \bar{v}_{p}\right)^{2} b \bar{n}_{i}(t)+\lambda_{m} \bar{c}_{i m}(t),
$$$$
q_{i}(t)=\sum_{i^{\prime}=i-1}^{i+1}\left[l_{i^{\prime}}(t) \bar{n}_{i^{\prime}}(t)-l_{i^{\prime}}(t) \bar{n}_{i}(t)\right]+S_{i}(t),
$$$$
Q_{i}(t)=l_{i+1 i}(t) \bar{n}_{i+1}(t)+l_{i-1 i}(t) \bar{n}_{i-1}(t)
$$$$
+\left[l_{i i+1}(t)+l_{i i-1}(t)\right] \bar{n}_{i}(t)+S_{i}(t),
$$

and

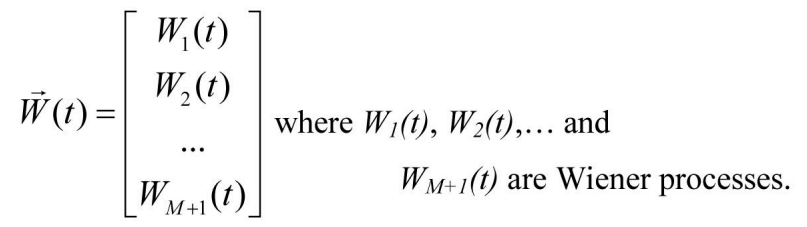

Eq. (6) is the stochastic space-dependent kinetics model (called the SSKM) for one spatial dimension, one energy cell, and $M$ delayed-neutron precursor groups. Recall that if $\hat{B}=0$, then Eq. (6) reduces to the FSM Eqs. (4) and (5), which, in turn, can reduce to the conventional point-kinetics equations as mentioned in the previous study. Hence, it is noted that the improved SSKM can also be considered as a space-dependent generalization of the stochastic point-kinetics equations $[10,12]$.

\section{NUMERICAL STUDIES}

Several typical numerical studies are performed using MATLAB and FORTRAN to validate the SSKM for $M$ precursor groups as well as to evaluate its applicability to more sophisticated reactor kinetics problems through comparison with analog Monte Carlo calculations. The numerical solution for Eq. (6) is similarly adopted from the previous study [10]. In the Monte Carlo procedure, the system is checked at each time step, to see if fission, decay, capture, or diffusion occurs or if a source neutron is produced. After all the trials have been generated, the mean and variance of the dynamic populations in the reactor are computed for comparison with the SSKM results.

The first example deals with six delayed-neutron precursor groups in which positive step reactivity is inserted into a homogeneous slab reactor. The second example analyzes the case of a heterogeneous slab reactor with uneven insertion of negative step reactivity. Finally, the variances in population dynamics are examined by adjusting the external source rate and initial conditions to determine how to keep the dynamic system under control from inherent random processes. This is very important in the safety analysis of reactor transients.

\subsection{Calculations for Six Delayed-neutron Precursor Groups}

The first example models positive step reactivity insertion within a homogeneous slab reactor which is partitioned into five space cells $(I=5)$. In this case, the values $\lambda_{m}=[0.0127,0.0317,0.115,0.311,1.4,3.87], \beta_{m}=$ $[0.000266,0.001491,0.001316,0.002849,0.000896$, $0.000182], \beta=0.007, v=2.5$, the fission rate $v \Sigma_{f}=20000 / \mathrm{sec}$, the absorption rate $v \Sigma_{a}=49850 / \mathrm{sec}$, the slab thickness $H$ $=150 \mathrm{~cm}$, the diffusion coefficient $D_{i}=0.046 \mathrm{~cm}$, and $v D_{i} B_{g i}^{2}=100 / \sec \left(B_{g i}^{2}=(\pi I / H)^{2}\right.$ is the geometric buckling for space cell $i$, where $i=1,2, \ldots, I ;[14])$ are considered (it is noted that $l_{i i^{\prime}}(t)=l_{i^{\prime}}(t)=\frac{v D_{i} B_{i i}^{2}}{2}$ for the uniform slab reactor of interest, where $\left.i^{\prime}=i-1, i+1\right)$. The uniform external source rate $S_{i}=6666.7 / \mathrm{sec}$ and the homogeneous initial conditions $\left[\bar{n}_{i}(0), \bar{c}_{i 1}(0), \ldots, \bar{c}_{i 6}(0)\right]=[33.3,34908.1,78391.2$, $19072.5,15268.0,1066.7,78.4]$ are adopted for each inner space cell $(i=2,3,4)$, while the vacuum boundary conditions are applied to the two outermost space cells $(i=1,5)$. Forty 0.1-second time intervals are used for the SSKM calculations. The number of trials for both the SSKM and Monte Carlo calculations is 5000, and it is found that the SSKM is more than one hundred times faster than the Monte Carlo method in this example.

As seen in Table 1, there is good agreement between the two different calculation procedures at time $t=0.1$ seconds. The mean values of neutron and precursor populations $\left(E\left(\bar{n}_{i}\right)\right.$ and $E\left(\Sigma_{m=1}^{6} \bar{c}_{i m}\right) ; i=1,2, \ldots, 5$ and $m=$ $1,2, \ldots, 6)$ are listed with their standard deviations $\left(\sigma\left(\bar{n}_{i}\right)\right.$ and $\sigma\left(\sum_{m=1}^{6} \bar{c}_{i m}\right) ; i=1,2, \ldots, 5$ and $\left.m=1,2, \ldots, 6\right)$. Figs. 1 
Table 1. A comparison of Mean Neutron Level and Mean Fission Product Level with Their Standard Deviations Calculated at Time $t=0.1$ Seconds using the SSKM and Monte Carlo Methods for Positive Step Reactivity Insertion within a Uniform Slab Reactor.

\begin{tabular}{|c|c|c|c|c|}
\hline Space cell number & Estimated values & Monte Carlo & SSKM & Relative difference $(\%)^{2}$ \\
\hline \multirow{4}{*}{1} & $E\left(\bar{n}_{1}(0.1)\right)$ & 0 & 0 & - \\
\hline & $\sigma\left(\bar{n}_{1}(0.1)\right)$ & - & - & - \\
\hline & $E\left(\sum_{m=1}^{6} \bar{c}_{1 m}(0.1)\right)$ & 0 & 0 & - \\
\hline & $\sigma\left(\sum_{m=1}^{6} \bar{c}_{1 m}(0.1)\right)$ & - & - & - \\
\hline \multirow{4}{*}{2} & $E\left(\bar{n}_{2}(0.1)\right)$ & 83.26 & 84.81 & $1.86 \%$ \\
\hline & $\sigma\left(\bar{n}_{2}(0.1)\right)$ & 101.30 & 78.70 & - \\
\hline & $E\left(\sum_{m=1}^{6} \bar{c}_{2 m}(0.1)\right)$ & 150400 & 150450 & $0.03 \%$ \\
\hline & $\sigma\left(\sum_{m=1}^{6} \bar{c}_{2 m}(0.1)\right)$ & 728.40 & 947.14 & - \\
\hline \multirow{4}{*}{3} & $E\left(\bar{n}_{3}(0.1)\right)$ & 93.82 & 93.84 & $0.02 \%$ \\
\hline & $\sigma\left(\bar{n}_{3}(0.1)\right)$ & 108.00 & 83.31 & - \\
\hline & $E\left(\Sigma_{m=1}^{6} \bar{c}_{3 m}(0.1)\right)$ & 150700 & 150880 & $0.12 \%$ \\
\hline & $\sigma\left(\sum_{m=1}^{6} \bar{c}_{3 m}(0.1)\right)$ & 761.40 & 1042.70 & - \\
\hline \multirow{4}{*}{4} & $E\left(\bar{n}_{4}(0.1)\right)$ & 81.96 & 82.52 & $0.68 \%$ \\
\hline & $\sigma\left(\bar{n}_{4}(0.1)\right)$ & 101.30 & 75.76 & - \\
\hline & $E\left(\sum_{m=1}^{6} \bar{c}_{4 m}(0.1)\right)$ & 150300 & 150450 & $0.08 \%$ \\
\hline & $\sigma\left(\sum_{m=1}^{6} \bar{c}_{4 m}(0.1)\right)$ & 720.80 & 943.20 & - \\
\hline \multirow{4}{*}{5} & $E\left(\bar{n}_{5}(0.1)\right)$ & 0 & 0 & - \\
\hline & $\sigma\left(\bar{n}_{5}(0.1)\right)$ & - & - & - \\
\hline & $E\left(\sum_{m=1}^{6} \bar{c}_{5 m}(0.1)\right)$ & 0 & 0 & - \\
\hline & $\sigma\left(\sum_{m=1}^{6} \bar{c}_{5 m}(0.1)\right)$ & - & - & - \\
\hline \multirow{2}{*}{ Total } & $\operatorname{Sum}\left(E\left(\bar{n}_{i}(0.1)\right)\right)$ & 259.04 & 261.17 & $0.82 \%$ \\
\hline & $\operatorname{Sum}\left(E\left(\sum_{m=1}^{6} \bar{c}_{i m}(0.1)\right)\right)$ & 451400 & 451780 & $0.08 \%$ \\
\hline
\end{tabular}

${ }^{a}$ Relative Difference $(\%)=100 *($ SSKM - Monte Carlo)/Monte Carlo

and 2 show the mean neutron and precursor populations for 5000 trials (i.e., 5000 sample paths or trajectories of the Wiener processes) as well as the neutron and precursor sample paths modeled by the SSKM.

\subsection{Calculations for Inhomogeneous Slab Reactor}

The second example simulates uneven insertion of negative step reactivity within a heterogeneous slab reactor which is divided into five space cells $(I=5)$. The spatial dependent model is given in Table 2 (note that $l_{i i}(t) \neq l_{i t}(t)$ for the inhomogeneous slab reactor of interest, where $i^{\prime}=$ $i-1, i+1)$ with the vacuum boundary conditions applied to the two outermost space cell $(i=1,5)$. Forty 0.01 -second time intervals are also used for SSKM calculations. The number of trials for both SSKM and Monte Carlo calculations is 5000. Table 3 denotes that the SSKM agrees well with the Monte Carlo method at time $t=0.01$ seconds.

\subsection{Investigation of the Variances in Population Dynamics}

It is noted that the Wiener process which governs the Itô stochastic differential equations varies without bound as time increases, whereas its mean always remain zero [13]. That is, the variances in population dynamics resulting from Eq. (6) may not be anticipated. For safety purposes, it is necessary to observe the variances of the 


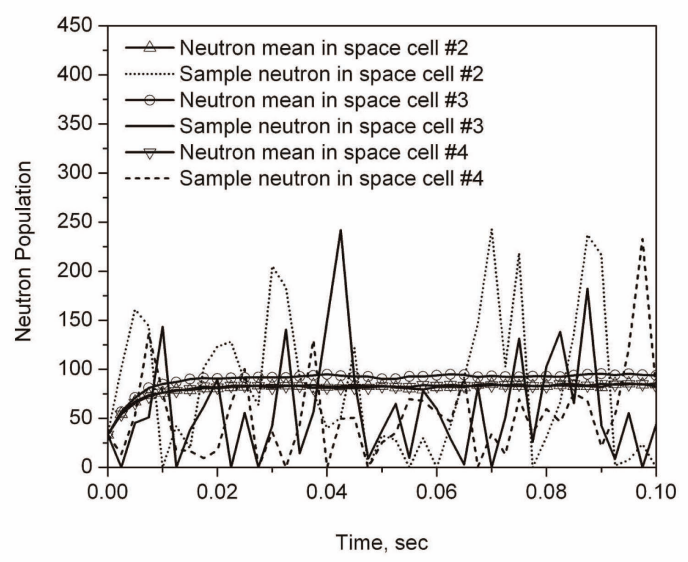

Fig. 1. Mean Neutron Level and One Neutron Sample Path for a Uniform Slab Reactor which is Partitioned into a Set of Space Cells.

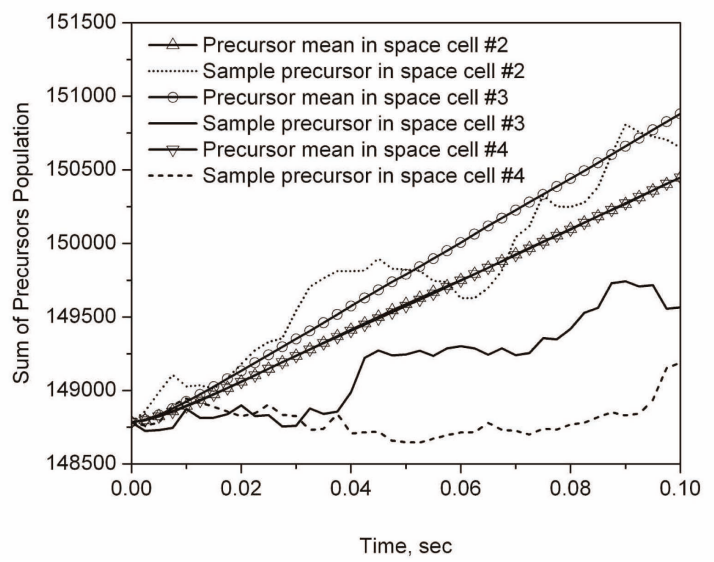

Fig. 2. Mean Precursor Level and One Precursor Sample Path for a Uniform Slab Reactor which is Partitioned into a Set of Space Cells.

Table 2. Spatial Dependent Model for a Heterogeneous Slab Reactor ( $\beta=0.0075, \lambda=0.075, \boldsymbol{v}_{p}=2.41, \boldsymbol{v}=220000 \mathrm{~cm} / \mathrm{sec}$ ).

\begin{tabular}{c|c|c|c}
\hline Kinetic parameters & Region 2 & Region 3 & Region 4 \\
\hline Fission rate & $v \Sigma_{f 2}=1920 / \mathrm{sec}$ & $v \Sigma_{f 3}=770 / \mathrm{sec}$ & $v \Sigma_{f 4}=1920 / \mathrm{sec}$ \\
\hline Absorption rate & $v \Sigma_{a 2}=4670 / \mathrm{sec}$ & $v \Sigma_{a 3}=1870 / \mathrm{sec}$ & $v \Sigma_{a 4}=4670 / \mathrm{sec}$ \\
\hline Slab thickness & $H_{2}=100 \mathrm{~cm}$ & $H_{3}=100 \mathrm{~cm}$ & $H_{4}=100 \mathrm{~cm}$ \\
\hline Diffusion coefficient & $D_{2}=0.15 \mathrm{~cm}$ & $D_{3}=0.09 \mathrm{~cm}$ & $D_{4}=0.15 \mathrm{~cm}$ \\
\hline External source & $S_{2}=500 / \mathrm{sec}$ & $S_{3}=300 / \mathrm{sec}$ & $S_{4}=500 / \mathrm{sec}$ \\
\hline Initial conditions & {$\left[\bar{n}_{2}(0), \bar{c}_{21}(0)\right]=[140,120]$} & {$\left[\bar{n}_{3}(0), \bar{c}_{31}(0)\right]=[140,120]$} & {$\left[\bar{n}_{4}(0), \bar{c}_{41}(0)\right]=[140,120]$} \\
\hline
\end{tabular}

neutron and precursor populations within the reactor by means of the external source rate and initial conditions for which the nature of the stochastic processes modeled by the SSKM can be clarified. In this fashion, the problem given in the Section 3.1 will be utilized here for illustration.

First, the source level is alternately increased by a factor of $10,50,100$, and 500 (called the source multiplication factor or $R_{E S}$ ), while the initial conditions are kept unchanged. Figs. 3 and 4 represent the ratios of the standard deviations to the mean values of neutron and precursor populations, i.e., the relative standard deviations in neutron and precursor distribution at $t=0.1$ seconds $\left(R_{S D N}\right.$ and $\left.R_{S D C}\right)$ as a function of the source multiplication factor $\left(R_{E S}\right)$. As shown in Fig. 3, the value of $R_{S D N}$ decreases as the source rate is increased. On the other hand, the $R_{S D C}$ behaves in an inverse manner in this region of $R_{E S}$. The reason seems to be that the increase in the source rate is not strong enough to depress the random fluctuations in the precursor population. Thus, the $R_{E S}$ still increases to the value of 20000 as seen in Fig. 4. This implies that the value of $R_{S D C}$ tends to decrease with sufficient increase of the source rate. Accordingly, it can be seen that the population dynamics are generally more stochastic at low source levels and tend to become less stochastic as the source rate is increased.

Secondly, the source level is maintained while the initial conditions are increased by a factor of $2,3, \ldots, 10$ (called the initial condition multiplication factor or $R_{I C}$ ). Figs. 5 and 6 show the relative standard deviations in neutron and precursor distribution at $t=0.1$ seconds $\left(R_{S D N}\right.$ and $\left.R_{S D C}\right)$ as a function of the initial condition multiplication factor $\left(R_{I C}\right)$. These figures show that both $R_{S D N}$ and $R_{S D C}$ decrease as the initial conditions are increased. In other words, the system of interest is found to behave more stochastically when it evolves from low initial conditions.

As a result, the random fluctuations in population dynamics can be made controllable by considering appropriate extraneous source level or initial conditions. In this case, the relative standard deviations in neutron and precursor distribution have a tendency to decrease with increasing source rate or initial conditions. 


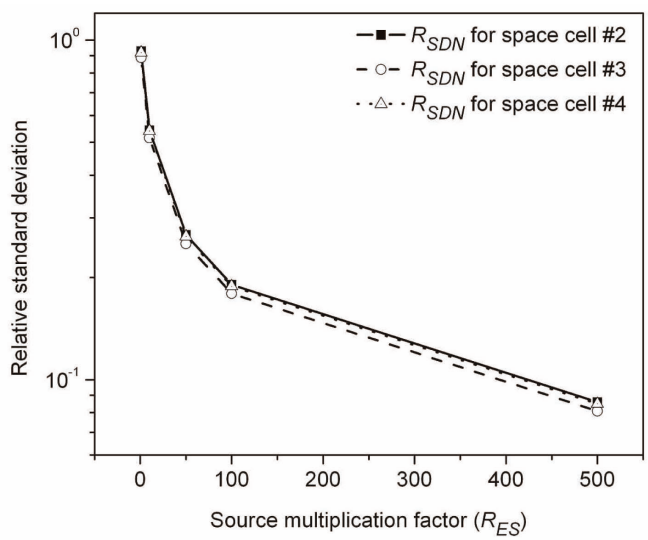

Fig. 3. The Relative Standard Deviation in Neutron Population at $t=0.1$ Seconds $\left(R_{S D N}\right)$ as a Function of the Source Multiplication Factor $\left(R_{E S}\right)$.

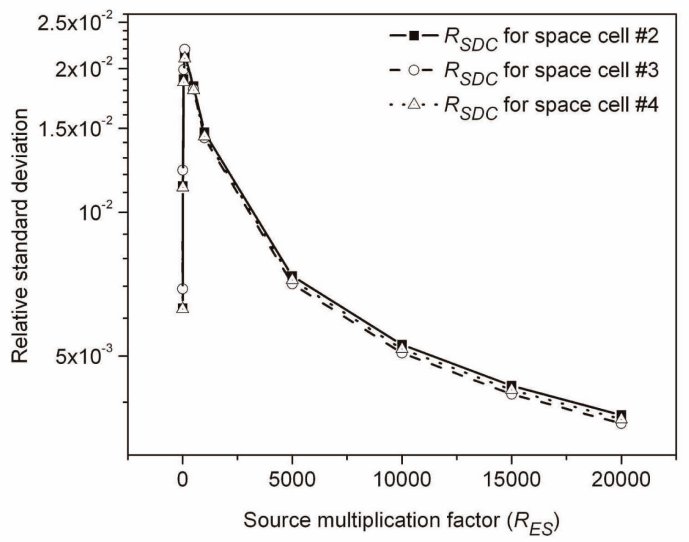

Fig. 4. The Relative Standard deviation in Precursor Population at $t=0.1$ Seconds $\left(R_{S D C}\right)$ as a Function of the Source Multiplication Factor $\left(R_{E S}\right)$.

Table 3. A comparison of Mean Neutron Level and Mean Fission Product Level with Their Standard Deviations Calculated at Time $t=0.01$ Seconds using the SSKM and Monte Carlo Methods for Uneven Insertion of Negative Step Reactivity within a Heterogeneous Slab Reactor.

\begin{tabular}{|c|c|c|c|c|}
\hline Space cell number & Estimated values & Monte Carlo & SSKM & Relative difference $(\%)^{a}$ \\
\hline \multirow{4}{*}{1} & $E\left(\bar{n}_{1}(0.1)\right)$ & 0 & 0 & - \\
\hline & $\sigma\left(\bar{n}_{1}(0.1)\right)$ & - & - & - \\
\hline & $E\left(\bar{c}_{11}(0.1)\right)$ & 0 & 0 & - \\
\hline & $\sigma\left(\bar{c}_{11}(0.1)\right)$ & - & - & - \\
\hline \multirow{4}{*}{2} & $E\left(\bar{n}_{2}(0.1)\right)$ & 78.99 & 78.60 & $-0.49 \%$ \\
\hline & $\sigma\left(\bar{n}_{2}(0.1)\right)$ & 50.20 & 58.03 & - \\
\hline & $E\left(\bar{c}_{21}(0.1)\right)$ & 156.81 & 156.73 & $-0.05 \%$ \\
\hline & $\sigma\left(\bar{c}_{21}(0.1)\right)$ & 12.16 & 14.75 & - \\
\hline \multirow{4}{*}{3} & $E\left(\bar{n}_{3}(0.1)\right)$ & 130.63 & 130.56 & $-0.05 \%$ \\
\hline & $\sigma\left(\bar{n}_{3}(0.1)\right)$ & 49.64 & 52.06 & - \\
\hline & $E\left(\bar{c}_{31}(0.1)\right)$ & 139.05 & 139.13 & $0.06 \%$ \\
\hline & $\sigma\left(\bar{c}_{31}(0.1)\right)$ & 4.55 & 4.91 & - \\
\hline \multirow{4}{*}{4} & $E\left(\bar{n}_{4}(0.1)\right)$ & 78.62 & 78.39 & $-0.29 \%$ \\
\hline & $\sigma\left(\bar{n}_{4}(0.1)\right)$ & 49.49 & 58.06 & - \\
\hline & $E\left(\bar{c}_{41}(0.1)\right)$ & 156.85 & 156.62 & $-0.15 \%$ \\
\hline & $\sigma\left(\bar{c}_{41}(0.1)\right)$ & 11.96 & 14.58 & - \\
\hline \multirow{4}{*}{5} & $E\left(\bar{n}_{5}(0.1)\right)$ & 0 & 0 & - \\
\hline & $\sigma\left(\bar{n}_{5}(0.1)\right)$ & - & - & - \\
\hline & $E\left(\bar{c}_{51}(0.1)\right)$ & 0 & 0 & - \\
\hline & $\sigma\left(\bar{c}_{51}(0.1)\right)$ & - & - & - \\
\hline \multirow{2}{*}{ Total } & $\operatorname{Sum}\left(E\left(\bar{n}_{i}(0.1)\right)\right)$ & 288.24 & 287.55 & $-0.24 \%$ \\
\hline & $\operatorname{Sum}\left(E\left(\bar{c}_{i 1}(0.1)\right)\right)$ & 452.71 & 452.48 & $-0.05 \%$ \\
\hline
\end{tabular}

${ }^{a}$ Relative Difference $(\%)=100 *(S S K M-$ Monte Carlo)/Monte Carlo 


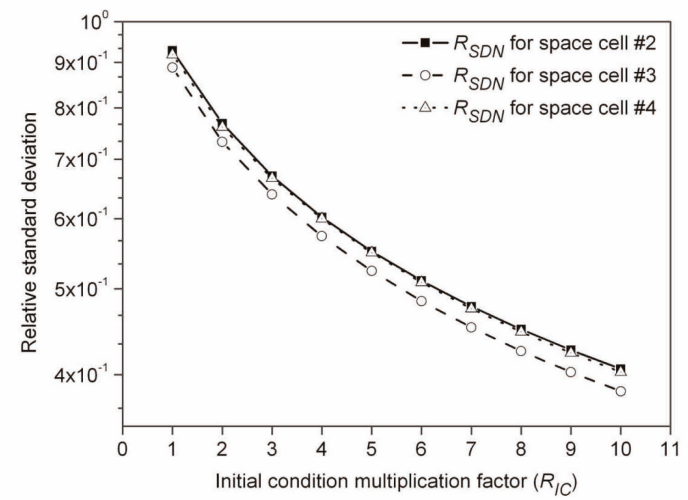

Fig. 5. The Relative Standard Deviation in Neutron Population at $t=0.1$ Seconds $\left(R_{S D N}\right)$ as a Function of the Initial Condition Multiplication Factor $\left(R_{I C}\right)$.

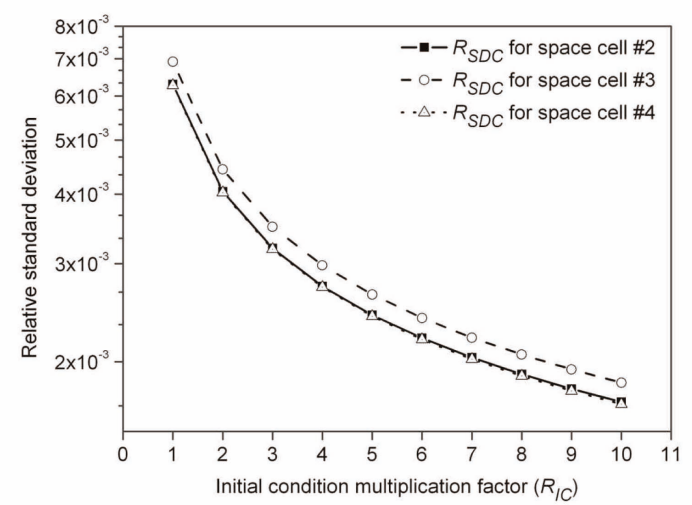

Fig. 6. The Relative Standard Deviation in Precursor Population at $t=0.1$ Seconds $\left(R_{S D C}\right)$ as a Function of the Initial Condition Multiplication Factor $\left(R_{I C}\right)$.

\section{CONCLUSIONS}

In this work, the stochastic space-dependent kinetics model (SSKM) was improved and tested with more realistic and complicated cases regarding six delayed-neutron precursor groups and inhomogeneous slab reactors where the reactivity or extraneous source can be introduced locally. The numerical studies showed that the improved SSKM agrees well with the Monte Carlo method. Again, the improved SSKM, which can be considered as a generalization of the stochastic point-kinetics equations, was found to provide a faster calculation method than Monte Carlo computation. In addition, the source level and the initial conditions were adjusted to investigate the random fluctuations of the neutron population and fission product levels across the reactor. Consequently, it was shown that the relative standard deviations in neutron and precursor populations tend to decrease with increasing source rate or initial conditions. That is, the random behavior of a nuclear reactor can be made controllable during transients. Thus, it is expected that the SSKM can be used for reactor transient analysis with possibility of controlling the variances of dynamic populations.

In future work, the SSKM may be further extended to handle few energy groups, and it will be applied to various practical problems involving space-time dependent reactor kinetics.

\section{ACKNOWLEDGEMENTS}

This study was supported by the Ministry of National Defense (2009-00000002197) and the Innovative Technology Center for Radiation Safety.

\section{REFERENCES}

[1] J. J. Duderstadt and L. J. Hamilton, Nuclear Reactor Analysis, John Wiley and Sons, New York, p. 233 (1976).

[2] D. L. Hetrick, Dynamics of Nuclear Reactors, The University of Chicago Press, Chicago (1971).

[3] Y. Ronen, CRC Handbook of Nuclear Reactors Calculations, Vol. II, p. 219, CRC Press, Florida (1986).

[4] W. M. Stacey, Space-Time Nuclear Reactor Kinetics, Academic Press, New York (1969).

[ 5 ] W. M. Stacey, Nuclear Reactor Physics, John Wiley and Sons, New York (2001).

[6] T. M. Sutton and B. N. Aviles, "Diffusion Theory Methods for Spatial Kinetics Calculations," Pro. Nucl. Energy, 30, 119 (1996).

[7] Jr. H. Hurwitz et al., "Kinetics of Low Source Reactor Startups Part I," Nucl. Sci. Eng., 15, 166 (1963).

[ 8 ] Jr. H. Hurwitz et al., "Kinetics of Low Source Reactor Startups Part II," Nucl. Sci. Eng., 15, 187 (1963).

[9] D. B. MacMillan and M. L. Storm, "Kinetics of Low Source Reactor Startups Part III," Nucl. Sci. Eng., 16, 369 (1963).

[10] P. N. V. Ha and J. K. Kim, "A Stochastic Approach to Monoenergetic Space-time Nuclear Reactor Kinetics," $J$. Nucl. Sci. Technol., 47, 705 (2010).

[11] E. J. Allen, Modeling with Itô Stochastic Differential Equations, Springer, New York (2007).

[12] J. G. Hayes and E. J. Allen, "Stochastic Point-kinetics Equations in Nuclear Reactor Dynamics," Ann. Nucl. Energy, 32, 572 (2005).

[13] P. E. Kloeden and E. Platen, Numerical Solution of Stochastic Differential Equations, Springer, New York (1995).

[14] J. R. Lamarsh and A. J. Baratta, Introduction to Nuclear Engineering, 3rd ed., p. 266, Prentice Hall, Upper Saddle River (2001) 